\section{Physical and Functional Interaction of Mitochondrial Single-Stranded DNA-Binding Protein and the Catalytic Subunit of DNA Polymerase Gamma}

\author{
Grzegorz L. Ciesielski ${ }^{1,2,3 *}$, Shalom Kim ${ }^{3}$, Carolina de Bovi Pontes ${ }^{3}$ and Laurie S. Kaguni ${ }^{1,2 *}$ \\ ${ }^{1}$ Department of Biochemistry and Molecular Biology and Center for Mitochondrial Science and Medicine, Michigan State \\ University, East Lansing, MI, United States, ${ }^{2}$ Institute of Biosciences and Medical Technology, University of Tampere, \\ Tampere, Finland, ${ }^{3}$ Department of Chemistry, Auburn University at Montgomery, Montgomery, AL, United States
}

The maintenance of the mitochondrial genome depends on a suite of nucleus-encoded proteins, among which the catalytic subunit of the mitochondrial replicative DNA polymerase, Pol $\gamma \alpha$, plays a pivotal role. Mutations in the Pol $\gamma \alpha$-encoding gene, POLG, are a major cause of human mitochondrial disorders. Here we present a study of direct and functional interactions of Pol $\gamma \alpha$ with the mitochondrial single-stranded DNA-binding protein (mtSSB). mtSSB coordinates the activity of the enzymes at the DNA replication fork. However, the mechanism of this functional relationship is elusive, and no direct interactions between the replicative factors have been identified to date. This contrasts strikingly with the extensive interactomes of SSB proteins identified in other homologous replication systems. Here we show for the first time that mtSSB binds Pol $\gamma \alpha$ directly, in a DNA-independent manner. This interaction is strengthened in the absence of the loop 2.3 structure in $\mathrm{mtSSB}$, and is abolished upon preincubation with Pol $\gamma \beta$. Together, our findings suggest that the interaction between mtSSB and polymerase gamma holoenzyme (Pol $\gamma$ ) involves a balance between attractive and repulsive affinities, which have distinct effects on DNA synthesis and exonucleolysis.

Keywords: mitochondrial DNA replication, DNA polymerase gamma, mitochondrial single-stranded DNA-binding protein, mitochondrial biogenesis, intermolecular interactions

Received: 07 June 2021 Accepted: 04 August 2021 Published: 01 September 2021

Citation:

Ciesielski GL, Kim S,

de Bovi Pontes $C$ and

Kaguni LS (2021) Physical and

Functional Interaction of Mitochondrial Single-Stranded DNA-Binding Protein and the Catalytic Subunit of DNA

Polymerase Gamma.

Front. Genet. 12:721864. doi: 10.3389/fgene.2021.721864

\section{INTRODUCTION}

Replication of the mitochondrial genome depends on a set of nucleus-encoded proteins (Ciesielski et al., 2016b). The synthesis of mitochondrial (mt)DNA is catalyzed by the DNA polymerase gamma holoenzyme (Pol $\gamma$ ), which in vertebrates comprises the catalytic subunit, Pol $\gamma \alpha$, and a dimeric accessory subunit, Pol $\gamma \beta_{2}$ (Kaguni, 2004; Ciesielski et al., 2016b). Notably, mutations in the nuclear POLG gene encoding Pol $\gamma \alpha$ are the most common cause of human mitochondrial diseases identified to date (Stumpf et al., 2013). We, and others, have demonstrated previously that the activity of Pol $\gamma$ is facilitated by 
mtSSB (Gray and Wong, 1992; Korhonen et al., 2004; Oliveira and Kaguni, 2011), which ensures optimal organization of the single-stranded (ss)DNA template (Ciesielski et al., 2015), and a maximal synthetic rate by Pol $\gamma$ (Cerrón et al., 2019).

The structural organization of mtSSB resembles that of bacterial SSB proteins, and it exhibits the same DNA binding modality (Morin et al., 2017; Oliveira and Ciesielski, 2021). However, whereas bacterial homologues have been demonstrated to play a direct role in the recruitment of the genome replication factors, no such physical interactions of mtSSB have been documented (Oliveira and Ciesielski, 2021). This appears to correspond with the lack of a large C-terminal domain in $\mathrm{mtSSB}$, which in the homologous SSB of Escherichia coli, as well as in cases of viral SSB proteins (e.g., phages T7 and T4), mediates the binding of replication factors (Curth et al., 1996; Raghunathan et al., 2000; Salinas and Benkovic, 2000; Hernandez and Richardson, 2019; Oliveira and Ciesielski, 2021). On the other hand, a recent real-time kinetic analysis suggested that a strong, specific interaction of $\mathrm{mtSSB}$ with Pol $\gamma$ is needed to dislodge the former from the DNA template, and ensure the maximal rate of DNA synthesis. In the absence of the putative interaction, the DNA synthesis rate of Pol $\gamma$ is significantly reduced (Cerrón et al., 2019). These results suggest that mtSSB may interact with replication factors directly, despite the lack of the C-terminus.

We have shown previously that the loop 2.3 structure of $\mathrm{mtSSB}$, which is conserved among vertebrates, is necessary for the stimulation of the activity of Pol $\gamma$ holoenzyme (Oliveira and Kaguni, 2011). Loop 2.3 of human mtSSB encompasses 12 amino acids, most of which are disordered in the crystal structure (Yang et al., 1997). The loop extrudes from the surface of the quaternary structure and carries a negative charge as indicated by electrostatic surface potential (Oliveira and Kaguni, 2011), resembling the character of the C-terminus of EcSSB. Our earlier structural predictions suggested that loop 2.3 might engage in a physical interaction with Pol $\gamma$ holoenzyme (Oliveira and Kaguni, 2011). In this study, we sought to evaluate a putative mtSSB-Pol $\gamma$ interaction and its relevance for DNA synthesis (Figure 1).

\section{MATERIALS AND METHODS}

Nucleotides, nucleic acids and proteins, available in the Supplementary Materials.

\section{Biolayer Interferometry}

Biolayer interferometry experiments were performed on Octet RED384 device (Fortebio). Streptavidin-sensors were hydrated in PBS for $10 \mathrm{~min}$ and the baseline was recorded for $1 \mathrm{~min}$. Sensors were next saturated with a ligand: $600 \mathrm{nM}$ BTN-mtSSB or BTN-mtSSB 12.3 , or $1 \mu \mathrm{M}$ BTN-ssDNA 40-oligomer (as indicated), for at least $5 \mathrm{~min}$. Functionalized sensors were blocked in Ac buffer: $50 \mathrm{mM}$ Tris- $\mathrm{HCl} \mathrm{pH}$

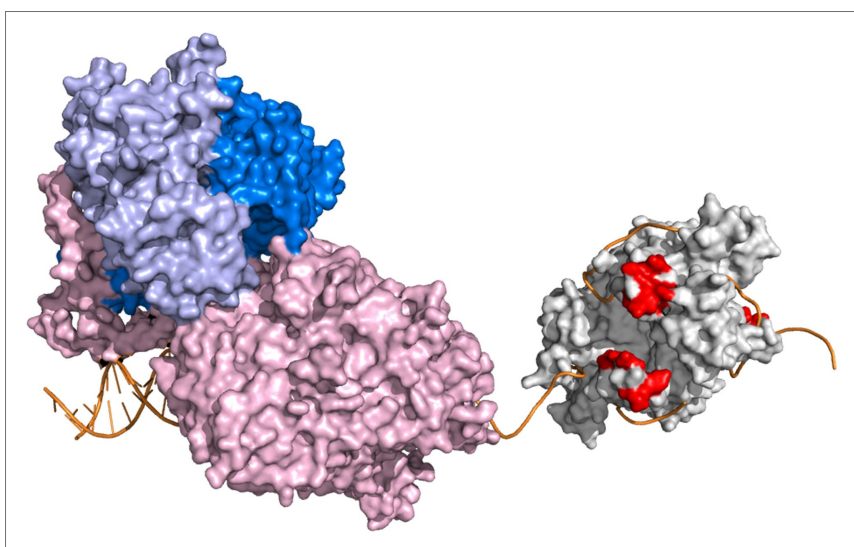

FIGURE 1 | Conceptual representation of DNA synthesis by Pol $\gamma$ on a mtSSB-bound ssDNA template. Pol $\gamma$ holoenzyme and mtSSB tetramer are represented by crystal structures PDB:4ZTZ (Szymanski et al., 2015) and PDB:6RUP (Piro-Mégy et al., 2019), respectively. The catalytic subunit, Pol $\gamma \alpha$, is represented in light pink. The dimeric accessory subunit, Pol $\gamma \beta_{2}$, is depicted in two shades of blue. The Pol $\gamma$-bound primer-template is oriented as in the original crystal structure. The mtSSB tetramer is represented in light grey. The acidic residues of the loop 2.3 structure of mtSSB (S67, D69, S70, E71, Y73, Q74, and D77) are shown in red. ssDNA was modeled onto mtSSB as described previously (Oliveira and Kaguni, 2011), by aligning ssDNA chains of the Escherichia coli SSB crystal structure (PDB:1EYG; Raghunathan et al., 2000) with the mtSSB tetramer structure. Orientation of mtSSB is dictated by the directionality of the modeled ssDNA. The software Pymol (www.pymol.org, Schrödinger, LLC) was used to create the figure. The scheme is not meant to detail structural and/or functional aspects of the replisome components.

8.0, $10 \mathrm{mM} \mathrm{KCl}, 4 \mathrm{mM} \mathrm{MgCl}_{2}, 0.4 \mathrm{mg} / \mathrm{ml} \mathrm{BSA}, 10 \mathrm{mM}$ DTT, for $5 \mathrm{~min}$. Association reactions were carried out subsequently in the Ac buffer with the addition of indicated analyte, for $10 \mathrm{~min}$. After that, sensors were placed back into the Ac buffer for $10 \mathrm{~min}$ to record dissociation. All the steps were performed at $30^{\circ} \mathrm{C}$ and $1,000 \mathrm{rpm}$ shaking rate. An exemplary experiment is represented in Figure 2A. The binding and dissociation curves were fitted to a 1:1 interaction model in case of the mtSSB-Pol $\gamma \alpha$ binding, and mass transfer model in case of ssDNA-mtSSB binding.

\section{Processive and Gap-Filling DNA Synthesis Assay}

The processive DNA synthesis assay (Figures 3A, 4A) was performed as described previously (Ciesielski et al., 2015). The calf-thymus DNA gap-filling assay (Figure 3B) was modified from (Oliveira and Kaguni, 2009). Twenty-five microliters reaction mixtures contained $50 \mathrm{mM}$ Tris- $\mathrm{HCl} \mathrm{pH} 8.5,4 \mathrm{mM}$ $\mathrm{MgCl}_{2}, 400 \mu \mathrm{g} / \mathrm{ml} \mathrm{BSA}, 10 \mathrm{mM}$ DTT, $30 \mathrm{mM} \mathrm{KCl}, 30 \mu \mathrm{M}$ (each) dNTPs mix, $\left[\alpha-{ }^{32} \mathrm{P}\right] \mathrm{dCTP}(2 \mu \mathrm{Ci}), 25 \mu \mathrm{g} / \mathrm{ml}$ DNase-I activated calf thymus DNA, $1 \mathrm{nM}$ Pol $\gamma \alpha$, and $850 \mathrm{nM} \mathrm{mtSSB}{ }_{4}$. The assay was carried out at $30^{\circ} \mathrm{C}$ for $10 \mathrm{~min}$. Samples were processed as described previously (Oliveira and Kaguni, 2009), and nucleotide incorporation was quantified in a liquid scintillation counter. 
A

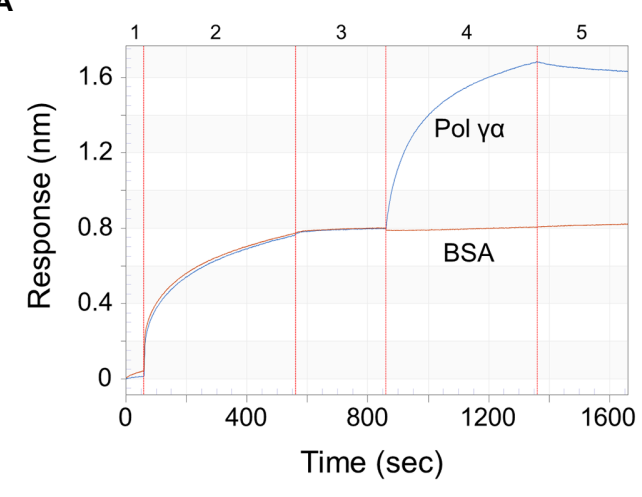

C

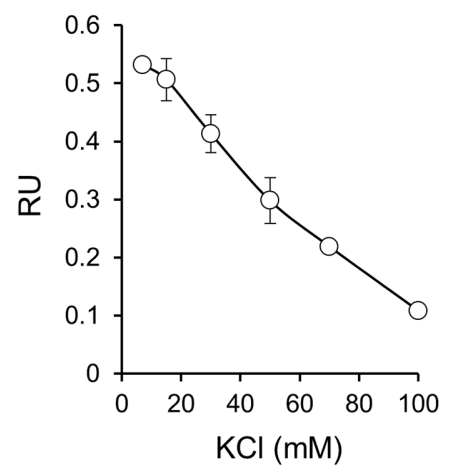

B

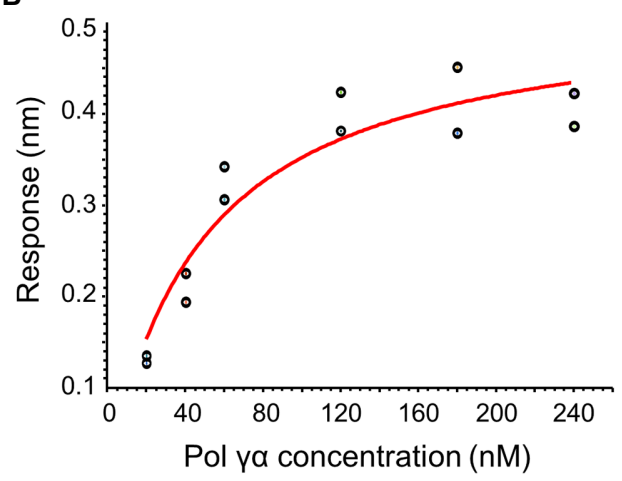

D
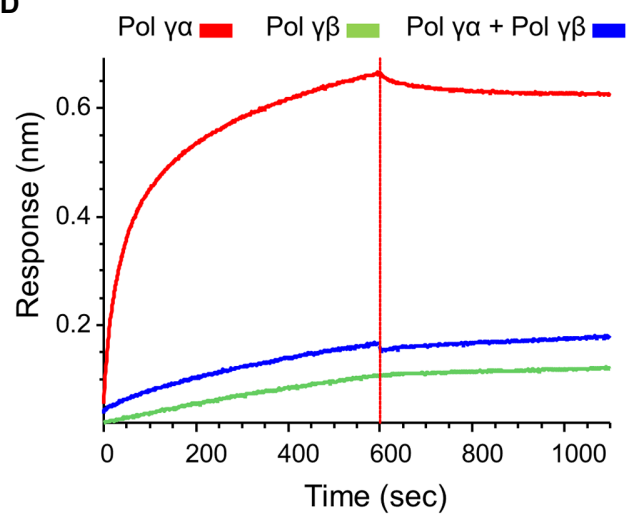

E

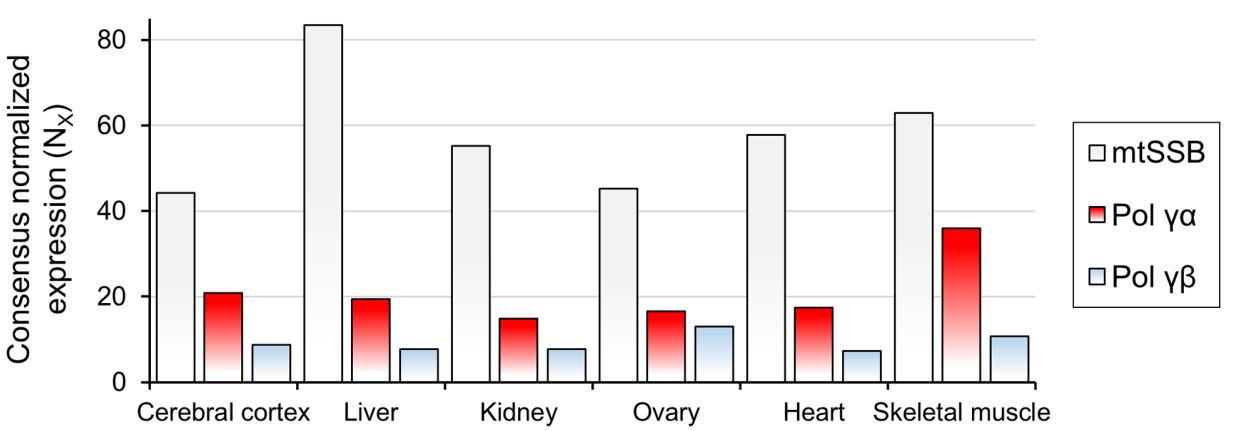

FIGURE 2 | mtSSB interacts physically with Pol $\gamma \alpha$ but not Pol $\gamma$ holoenzyme. (A) The affinity of mtSSB for Pol $\gamma \alpha$ was assessed by biolayer interferometry (BLI), as described under Materials and Methods. A representative sensogram is shown, and the numbers atop indicate individual experimental steps. Briefly, hydrated biosensors (1) were saturated with mtSSB (2), blocked with BSA (3), and placed into solutions of Pol $\gamma \alpha$. The association of the proteins was measured in real-time for $5 \mathrm{~min}$ (4), after which the sensors were moved to a buffer to allow dissociation (5). Excess BSA was present in all solutions used in steps $3-5$. (B) The binding affinity of mtSSB for Pol $\gamma \alpha$ was determined by BLI, as described in (A), using two sets of association responses upon increasing Pol $\gamma \alpha$ concentrations (indicated), fitted to a 1:1 binding model (see also Supplementary Figure 2). (C) The effect of salt concentration on the stability of the Pol $\gamma \alpha$-mtSSB complex was assessed by measuring changes in the association response of $120 \mathrm{nM} \mathrm{Pol} \gamma \alpha$ in the presence of increasing $\mathrm{KCl}$ concentrations. (D) The association of Pol $\gamma \beta$ with mtSSB, and its effect on the mtSSB-Pol $\gamma \alpha$ binding, was assessed as described in (A), replacing or preincubating $120 \mathrm{nM}$ Pol $\gamma \alpha$ with $120 \mathrm{nM}$ Pol $\gamma \beta_{2}$, as indicated. (E) The data representing the consensus transcript expression values $\left(N_{x}\right)$ of Pol $\gamma \alpha$, Pol $\gamma \beta$, and mtSSB in selected tissues, were obtained from Human Protein Atlas. Pol $\gamma \alpha$ corresponds to entry POLG (https://www.proteinatlas.org/ENSG00000140521-POLG/tissue); Pol $\gamma \beta$ to entry POLG2 (https://www.proteinatlas.org/ ENSG00000256525-POLG2/tissue); mtSSB to SSBP1 (https://www.proteinatlas.org/ENSG00000106028-SSBP1/tissue). The Consensus Normalized eXpression (NX) levels were created by the Human Protein Atlas team, by combining the data from the three transcriptomics datasets (HPA, GTEx, and FANTOM5) using the internal normalization pipeline.

\section{Processivity Assay}

The processivity assay (Figure $\mathbf{4 B}$ ) is a modification of the processive DNA-synthesis assay. Reaction mixtures of $20 \mu \mathrm{l}$ total volume contained $50 \mathrm{mM}$ Tris- $\mathrm{HCl} \mathrm{pH} 8.5,4 \mathrm{mM} \mathrm{MgCl}_{2}$, $400 \mu \mathrm{g} / \mathrm{ml} \mathrm{BSA}, 10 \mathrm{mM} \mathrm{DTT}, 30 \mathrm{mM} \mathrm{KCl}, 1 \mathrm{mM}$ mix of
dGTP, dTTP, dCTP, $0.5 \mathrm{mM}$ dATP, $1 \mathrm{mM}$ Cy3-dUTP, $5 \mathrm{nM}$ of singly-primed M13 DNA, $5 \mathrm{nM}$ Pol $\gamma \alpha$, and $425 \mathrm{nM}$ or $850 \mathrm{nM}$ of $\mathrm{mtSSB}_{4}$ variant. Assays were carried out at $37^{\circ} \mathrm{C}$ for $30 \mathrm{~min}$ and analyzed on $6 \%$ denaturing ( $7 \mathrm{M}$ Urea) polyacrylamide gels. 


\section{mtSSB Displacement Assay}

The mtSSB displacement was inferred from the efficiency of primer extension (Figure 4D) carried out in a $20 \mu$ l reaction mixture containing $50 \mathrm{mM}$ Tris $\mathrm{HCl} \mathrm{pH} \mathrm{8.5,20} \mathrm{mM} \mathrm{KCl,} 10 \mathrm{mM}$ DTT, $4 \mathrm{mM} \mathrm{MgCl}_{2}, 0.1 \mathrm{mg} / \mathrm{ml} \mathrm{BSA}, 10 \%$ glycerol, $100 \mu \mathrm{M}$ (each) dNTPs mix, $20 \mathrm{nM}$ fluorescently-labeled primer-template (15/59) DNA substrate, $5 \mathrm{nM} \mathrm{Pol} \gamma \alpha$, and $20 \mathrm{nM}$ or $60 \mathrm{nM}$ of $\mathrm{mtSSB}_{4}$ variant. Assays were performed at $37^{\circ} \mathrm{C}$ for $30 \mathrm{~min}$, and products were analyzed by native $12 \%$ polyacrylamide electrophoresis.

\section{Single Gap and Exonuclease Assays}

The single gap and exonuclease assays were carried out under the same conditions as the mtSSB displacement assay, except that fluorescently-labeled single gap DNA substrates indicated in Figures 3C,D, were used. The single gap assay was carried out in the presence or absence of 10 or $20 \mathrm{nM} \mathrm{mtSSB}{ }_{4}$, for $10 \mathrm{~min}$. The exonuclease assay was performed in the absence of dNTPs in the reaction mixture, in the presence or absence of $20 \mathrm{nM} \mathrm{mtSSB}$ or $\mathrm{mtSSB}_{12.3}$, for $30 \mathrm{~min}$. Where indicated, Pol $\gamma$ holoenzyme was used instead of Pol $\gamma \alpha$, at equivalent concentration. Samples were analyzed by denaturing $18 \%$ polyacrylamide gel electrophoresis.

\section{RESULTS}

\section{Direct Interaction Between mtSSB and Pol $\gamma \alpha$ Is Abolished by the Pol $\gamma \beta$ Subunit}

We examined the putative physical interaction between Pol $\gamma$ and $\mathrm{mtSSB}$ by biolayer interferometry (Ciesielski et al., 2016a), using purified recombinant proteins. To utilize mtSSB as the ligand, we conjugated it with biotin (see Materials and Methods; Supplementary Figure 1A), and documented that the modification did not affect its ability to stimulate Pol $\gamma$ activity in vitro (Supplementary Figure 1B). Then, streptavidin-coated biosensors were saturated with biotinylated (BTN-)mtSSB and used to assess the affinity of analytes in real-time (see Materials and Methods for details). We observed that Pol $\gamma \alpha$ binds to $\mathrm{mtSSB}$-sensors with a dissociation constant of $\mathrm{K}_{\mathrm{D}}=48 \mathrm{nM}$ $( \pm 9.9 \mathrm{nM}$; Figures $2 \mathrm{~A}, \mathbf{B})$. The specificity of the binding can be inferred from the fact that it occurs in spite of the presence of excess bovine serum albumin (BSA), which alone exhibited no affinity for the functionalized sensors (Figure 2A). The physical mtSSB-Pol $\gamma \alpha$ interaction was shown to be electrostatic in nature, as increasing salt concentration eliminates it (Figure 2C). In contrast to Pol $\gamma \alpha$, we observed that Pol $\gamma \beta$ does not bind to mtSSB-sensors under our experimental conditions (Figure 2D). Moreover, preincubation of Pol $\gamma \alpha$ with Pol $\gamma \beta$ at a $1 \alpha: 1 \beta_{2}$ molar ratio abolished the Pol $\gamma \alpha-\mathrm{mtSSB}$ binding (Figure 2D).

In the context of the mtDNA replication, functional interaction between Pol $\gamma$ and mtSSB occurs at the DNA template. We tested whether the presence of ssDNA affects the observed interaction. To that end, mtSSB-sensors were incubated in solutions containing increments of 43-nt-long ssDNA oligomers, and then placed into the Pol $\gamma \alpha$ solution; we observed no effect of pre-binding of ssDNA on the capacity of $\mathrm{mtSSB}$ to bind Pol $\gamma \alpha$ (Supplementary Figures 2, 3).

\section{mtSSB Stimulates the Activity of Pol $\gamma \alpha$ by Directing It to the Primer Site}

While the cooperative function of the Pol $\gamma \alpha$ and Pol $\gamma \beta$ subunits is pivotal for mtDNA replication, comprehensive transcriptomics analysis, presented in the Human Protein Atlas ${ }^{1}$ (Uhlen et al., 2015, 2017; Thul et al., 2017), demonstrates that in the majority of tissues, Pol $\gamma \alpha$ is expressed to a greater extent than Pol $\gamma \beta$ (roughly $\sim 2$-fold, Figure 2E). This implies that Pol $\gamma \alpha$, and its interaction with $\mathrm{mtSSB}$, may have an independent physiological role.

To assess the functional relevance of the mtSSB-Pol $\gamma \alpha$ interaction, we first tested the efficiency of processive DNA synthesis by Pol $\gamma \alpha$ on a singly-primed M13 ssDNA template, in the presence of increments of mtSSB (Figure 3A). We observed that at concentrations lower than that needed for DNA template saturation, mtSSB stimulates modestly DNA synthesis by Pol $\gamma \alpha$. At concentrations approximating that needed to saturate the ssDNA template, mtSSB inhibited Pol $\gamma \alpha$ activity. In contrast, Pol $\gamma$ holoenzyme was significantly more efficient and its activity was stimulated when levels of mtSSB exceeded that required for template saturation, in agreement with our previous report (Ciesielski et al., 2015). These observations indicate that the stimulatory effect of mtSSB on Pol $\gamma \alpha$ likely results from limiting the binding of Pol $\gamma \alpha$ to ssDNA, effectively directing Pol $\gamma \alpha$ to the primer site. The inhibition of Pol $\gamma \alpha$ at higher mtSSB concentrations suggests that, in contrast to the holoenzyme, Pol $\gamma \alpha$ exhibits limited ability to displace mtSSB from the DNA template.

It has been previously suggested that Pol $\gamma \alpha$ may be engaged in gap-filling during mtDNA repair (Longley et al., 1998a; Pinz and Bogenhagen, 2006; Kazak et al., 2012). In support of this, Pol $\gamma \alpha$ exhibits a relatively high processivity of $\sim 100 \mathrm{nt}$ (Longley et al., 1998b; Johnson et al., 2000), as compared to that of $1-15 \mathrm{nt}$ of other DNA polymerase catalytic subunits (McHenry and Kornberg, 1977; Hori et al., 1979; Lee et al., 2010), and exhibits higher fidelity than Pol $\gamma$ holoenzyme (Longley et al., 2001). To test whether the interaction with mtSSB might facilitate the gap-filling activity of Pol $\gamma \alpha$, we tested the nucleotide incorporation rate of Pol $\gamma \alpha$ on DNase I-activated calf-thymus (ct)DNA, which contains nicks, short ssDNA gaps, and an overall high primer density (Wernette et al., 1988), in the presence and absence of mtSSB (Figure 3B). We found that the presence of $\mathrm{mtSSB}$ resulted in an $\sim 8$-fold increase in nucleotide incorporation by Pol $\gamma \alpha$. To evaluate in detail the role of mtSSB in the gap-filling activity of Pol $\gamma \alpha$, we performed a primer-extension assay using DNA substrates containing either a 5 or $35 \mathrm{nt}$ gap, with or without a downstream 5 'flap (Figure 3C). The $35 \mathrm{nt}$ gap as well as the $40 \mathrm{nt}$ 5'-flap were designed to allow binding of a single mtSSB molecule, in accordance with its DNA-binding size under the experimental conditions used (i.e., at low salt; Morin et al., 2017). We observed

${ }^{1}$ http://www.proteinatlas.org 
A
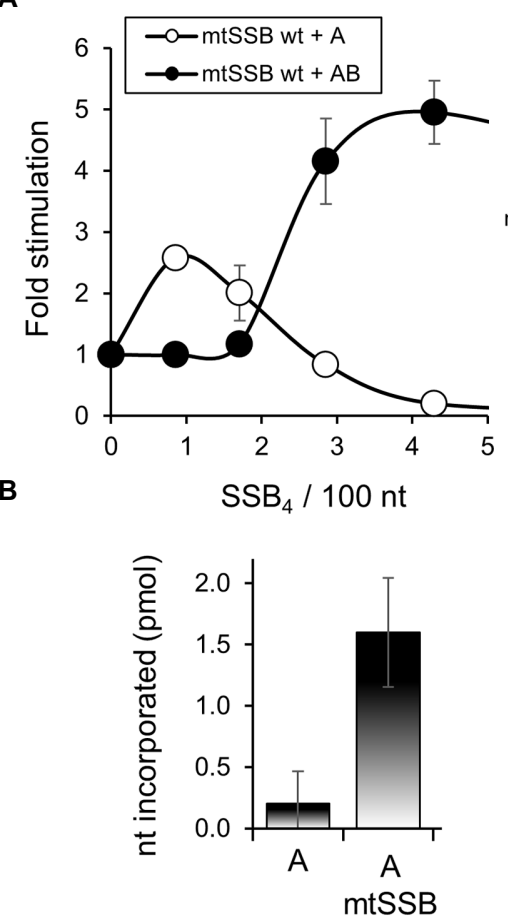

C

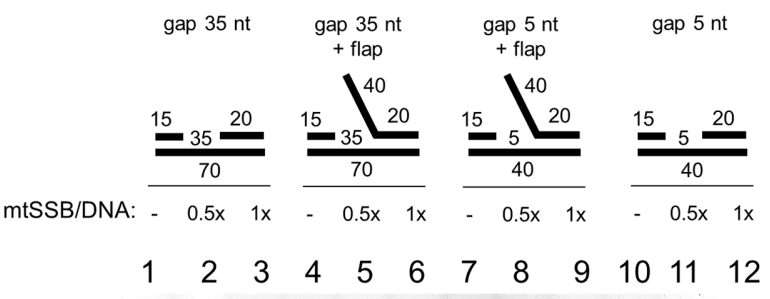

D
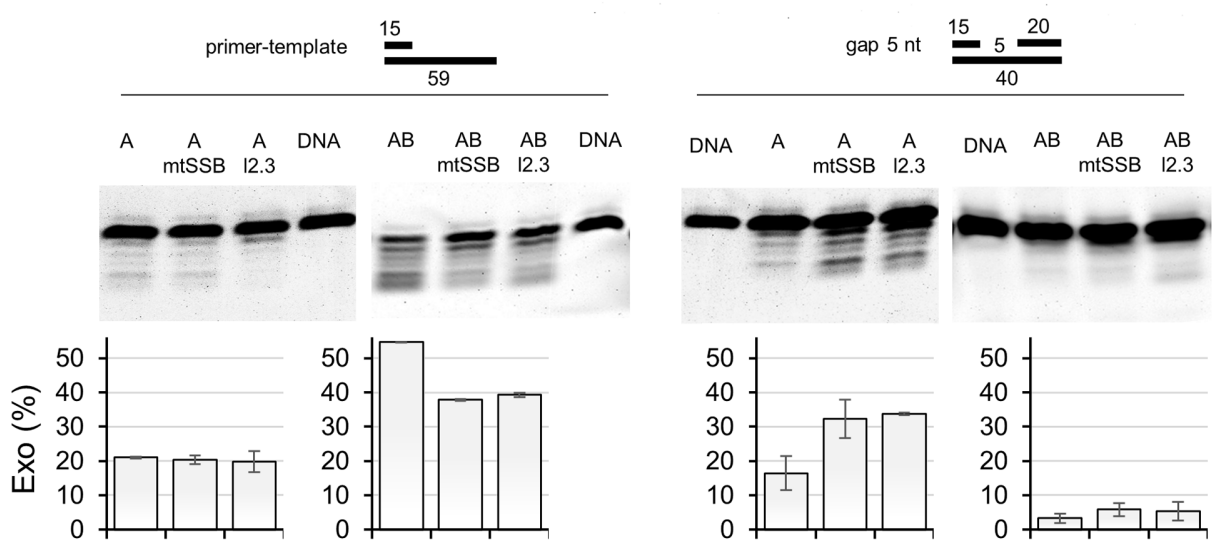

FIGURE 3 | mtSSB stimulates the activity of Pol $\gamma \alpha$. (A) The processive DNA synthesis assay was performed as described under Materials and Methods, using $55 \mathrm{fmol}$ singly-primed M13 circular DNA (6,407 nt), $20 \mathrm{fmol}$ of Pol $\gamma \alpha$ (A, open circles) or Pol $\gamma$ holoenzyme (AB, closed circles) and increasing amounts of mtSSB: $0,3,6,10$, and $15 \mathrm{pmol}$. Assays were performed at $30 \mathrm{mM} \mathrm{KCl}$ and $4 \mathrm{mM} \mathrm{MgCl}$. The data represent the mean of three experiments, $\pm S D$. The results were normalized to the amount of nucleotide incorporated by Pol $\gamma \alpha$ or Pol $\gamma$ holoenzyme in the absence of mtSSB (arbitrarily set to 1 in each case). (B) Nucleotide incorporation on DNase I-activated calf thymus DNA was assessed as described under Materials and Methods, using Pol $\gamma \alpha(\mathrm{A})$, in the presence or absence of mtSSB, as indicated. The data represent the mean of three experiments, \pm SD. (C) The single gap assay was performed as described under Materials and Methods in the absence or presence of 10 or $20 \mathrm{nM} \mathrm{mtSSB}$, represented as their molar ratio to available binding sites on the DNA substrate (mtSSB/DNA). DNA substrates with a 5 or $35 \mathrm{nt}$ gap, with or without a $40 \mathrm{nt}$ flap were used as indicated. Products of reactions were analyzed by denaturing polyacrylamide gel electrophoresis. The gel image is representative of three independent analyses. (D) The exonuclease assay was performed as described under Materials and Methods, using Pol $\gamma \alpha(\mathrm{A})$ or Pol $\gamma$ holoenzyme (AB), in the presence or absence of $20 \mathrm{nM}_{\mathrm{mtSSB}} \mathrm{or}_{\mathrm{mtSSB}} \mathrm{B}_{12 .}$. DNA substrate with a $5 \mathrm{nt}$ gap, or primer-template (15/59) were used as indicated. Products of reactions were analyzed by denaturing polyacrylamide gel electrophoresis. The relative abundance of the products of exonucleolysis was estimated by densitometry and the mean of three independent experiments $( \pm \mathrm{SD})$ is presented in the graphs below.

no qualitative or quantitative differences between DNA products generated by Pol $\gamma \alpha$ in the presence or absence of mtSSB on any of the DNA substrates used (Figure 3C). This indicates that the interaction of mtSSB with Pol $\gamma \alpha$ does not affect the gap-filling activity directly, and the increase in activity in the ctDNA assay likely resulted from restricting the non-productive binding of Pol $\gamma \alpha$ to ssDNA stretches, as in the case of the processive DNA synthesis assay. This analysis also showed that 
the interaction does not promote the strand-displacement DNA synthesis, as in the absence of the 5'-flap, Pol $\gamma \alpha$ synthesized products only $1 \mathrm{nt}$ longer than the gap size, which corroborates a previous report showing that Pol $\gamma \alpha$ has a modest ability to melt the DNA duplex (He et al., 2013). Here we show, however, that this ability is counteracted by the presence of the 5'-flap. In addition, we observed no apparent reduction in the quantity of the products generated over the $35 \mathrm{nt}$ gap in the presence of $\mathrm{mtSSB}$, which suggests that Pol $\gamma \alpha$ alone is able to displace a single mtSSB from the DNA template.

In addition to its polymerase activity, Pol $\gamma \alpha$ is capable of exonucleolytic activity, which in addition to its role in proofreading during DNA replication, may also be relevant for excision-based mtDNA repair. Notably, excision of certain lesions, such as 8-oxoguanine, can be catalyzed by Pol $\gamma \alpha$ only in the absence of Pol $\gamma \beta$ (Wong et al., 2009). To assess whether the interaction with $\mathrm{mtSSB}$ may facilitate the exonucleolytic activity of Pol $\gamma \alpha$, we tested primer excision in the presence and absence of mtSSB, using a DNA substrate containing a $5 \mathrm{nt}$ gap (Figure 3D). We observed that $\mathrm{mtSSB}$ stimulated primer excision, which considering the lack of mtSSB binding sites on this DNA substrate, can be explained by a direct participation of $\mathrm{mtSSB}$ in Pol $\gamma \alpha$ loading. To test this, we repeated the experiment in the presence of Pol $\gamma \beta$. We observed that the Pol $\gamma$ holoenzyme was virtually incapable of excising the primer on this substrate, and the presence of mtSSB did not affect this. This indicates that an interruption of the Pol $\gamma \alpha$-mtSSB interaction by the stronger competitor impedes utilization of the gapped substrate by $\operatorname{Pol} \gamma \alpha$, hence supporting the relevance of the interaction for loading of Pol $\gamma \alpha$ onto short gap sites. In addition, we performed the exonuclease assay using a primertemplate substrate (Figure 3D). Notably, in this case, the exonucleolytic activity of the holoenzyme was much greater than that of Pol $\gamma \alpha$, which is consistent with previous reports (Gray and Wong, 1992). mtSSB had no effect on the exonucleolytic activity of Pol $\gamma \alpha$ in this case, whereas it was inhibitory to the activity of the holoenzyme.

\section{Loop 2.3 Weakens the Ability of mtSSB to Interact Directly With Pol $\gamma \alpha$ and the DNA Template}

We reported previously that a variant of mtSSB lacking nine residues of loop 2.3 (i.e., $\Delta 67-75$ ), here denoted as $\mathrm{mtSSB}_{12.3}$, fails to stimulate processive DNA synthesis by Pol $\gamma$ holoenzyme (Oliveira and Kaguni, 2011; Ciesielski et al., 2015; Figure 4A). Here we tested whether this also applies to the activity of the Pol $\gamma \alpha$ subunit. We observed that, in contrast to Pol $\gamma$ holoenzyme, $\mathrm{mtSSB}_{12.3}$ stimulated the activity of Pol $\gamma \alpha$. Moreover, the stimulatory effect of $\mathrm{mtSSB}_{12.3}$ was 3 -4-fold greater than in the case of the wild-type protein (Figure 4A), approaching the nucleotide incorporation by Pol $\gamma$ holoenzyme under these conditions (Supplementary Figure 4). This suggested to us that the processivity of Pol $\gamma \alpha$ is enhanced in the presence of $\mathrm{mtSSB}_{12.3}$. Therefore, we tested the extent of primer elongation by Pol $\gamma \alpha$ on the M13 ssDNA template, in the presence or absence of the mtSSB variant. We found no difference in the length of products generated by Pol $\gamma \alpha$, although shorter species became more apparent when $\mathrm{mSSSB}_{12.3}$ was present in the reaction (Figure $\mathbf{4 B}$, bold arrow). This indicates that the elevated stimulatory effect of $\mathrm{mtSSB}_{12.3}$ on the activity of Pol $\gamma \alpha$ does not result from the enhanced processivity of the enzyme.

It was suggested previously that loop 2.3 facilitates a repulsive interaction of $\mathrm{mtSSB}$ with Pol $\gamma$, enabling the displacement of the former from the template (Cerrón et al., 2019). We tested whether the lack of loop 2.3 affects the Pol $\gamma \alpha$-mtSSB interaction by biolayer interferometry, using sensors saturated with $\mathrm{BTN}-\mathrm{mtSSB}_{12.3}$. We found that Pol $\gamma \alpha$ binds to $\mathrm{mtSSB}_{12.3}$ with a $K_{D}=26 \mathrm{nM}( \pm 4.9 \mathrm{nM}$; Figure $4 \mathrm{C})$, which is approximately 2 -fold stronger than in the case of the wild-type mtSSB. To test whether loop 2.3 is relevant for the displacement of mtSSB from the DNA template, we examined the efficiency of primer extension over a stretch of $44 \mathrm{nt}$, in the presence or absence of wild-type versus loop 2.3-deficient mtSSB (Figure 4D). Consistent with the earlier results (Figures 3A,C), the wildtype mtSSB did not affect the activity of Pol $\gamma \alpha$ when used at 1:1 molar ratio to DNA template, but reduced its activity slightly $(\sim 10 \%)$ only when used at 3 -fold excess. In contrast, $\mathrm{mtSSB}_{12.3}$ inhibited primer extension by $\sim 10 \%$ at $1: 1$ molar ratio to DNA template, and by $\sim 25 \%$, at the three-fold excess. These results indicate that while Pol $\gamma \alpha$ can displace the wildtype mtSSB to some extent, the lack of loop 2.3 impedes this activity and consequently, limits DNA synthesis.

The reduced capacity of Pol $\gamma$ to displace $\mathrm{mtSSB}_{12.3}$ may result from an elevated DNA-binding affinity of the latter, which we suggested previously (Oliveira and Kaguni, 2011). To evaluate this, we compared the ssDNA-binding affinity of the wild-type and loop2.3-deficient mtSSB by biolayer interferometry, using sensors saturated with a 40 nt-long ssDNA oligomer. In both cases, the binding traces indicated a non-1:1 binding, likely due to binding cooperativity and multivalency of both the ligand and the analyte (Supplementary Figure 5). In addition, mass transfer interference was evident. Because of these limitations we chose to estimate DNA-binding affinities from the equilibrium responses of best fitted curves. We found a $K_{D}$ of $2.3 \mathrm{nM}$ for the wild-type $\mathrm{mtSSB}$, and $1.4 \mathrm{nM}$ for $\mathrm{mtSSB}_{12.3}$ (Figure $4 \mathrm{E}$ ). This indicates that the lack of loop 2.3 enhances ssDNA binding by $\mathrm{mtSSB}$. Notably, these values, as well as the resulting difference between them, are in very good agreement with our earlier estimations by gel mobility shift assay of $3.8 \mathrm{nM}$ for wild-type $\mathrm{mtSSB}$, and $2.4 \mathrm{nM}$ for $\mathrm{mtSSB}_{12.3}$ (Oliveira and Kaguni, 2011).

Finally, we assessed the effect of the loop 2.3-deficient mtSSB on the exonucleolytic activity of Pol $\gamma \alpha$ (Figure 3D). We observed that in the absence of $\mathrm{mtSSB}$ binding sites ( $5 \mathrm{nt}$ gap), $\mathrm{mtSSB}_{12.3}$ stimulated primer excision by Pol $\gamma \alpha$ to the same extent as the wild-type protein, whereas it had no effect on the activity of the holoenzyme. This agrees with our interpretation that the attraction between the proteins contributes to loading of Pol $\gamma \alpha$ onto short gaps. When $\mathrm{mtSSB}_{\mathrm{l2.3}}$ was able to bind to the DNA substrate (primer-template), the exonucleolytic processivity of Pol $\gamma \alpha$ was reduced to approximately a single nucleotide, while the overall abundance of the products remained similar to that generated by the wild-type mtSSB, or Pol $\gamma \alpha$ alone. 
A

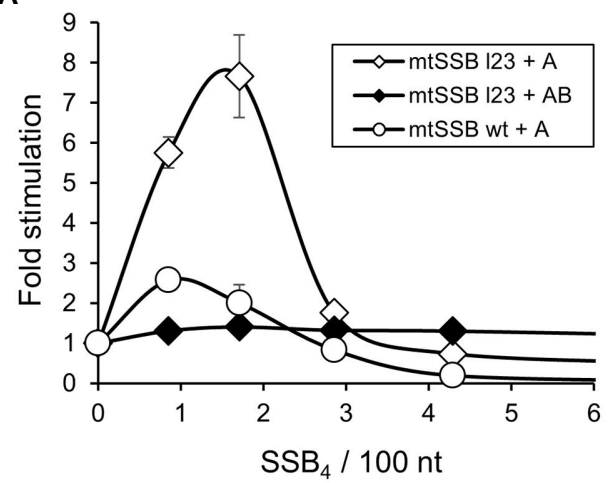

B

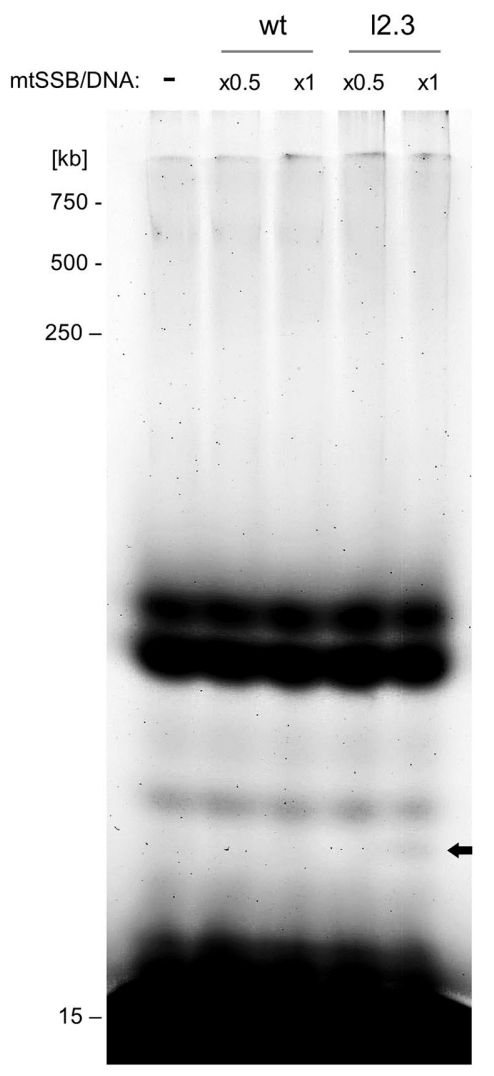

C

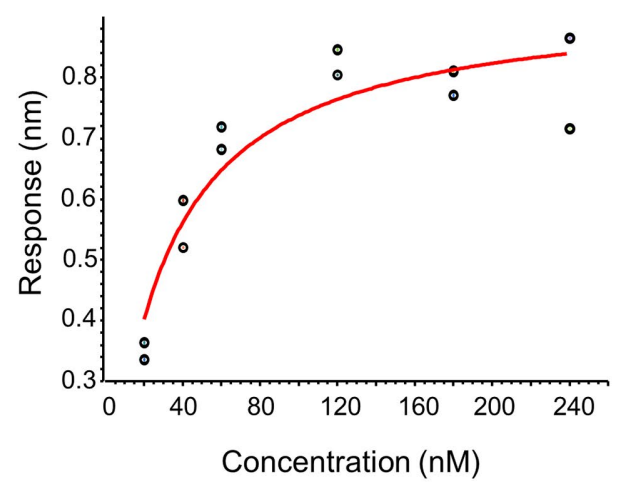

D

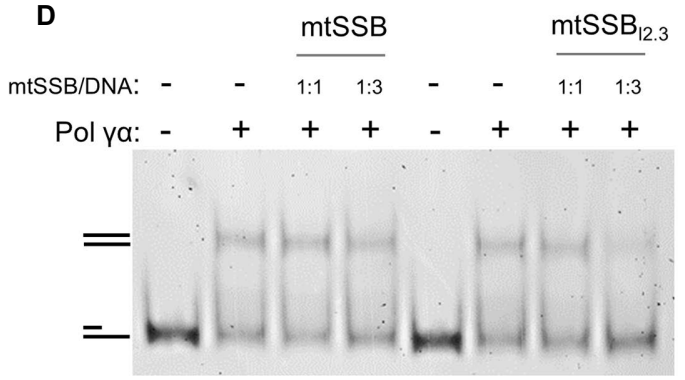

[\% product]

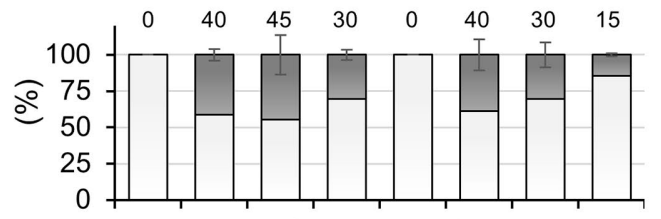

E

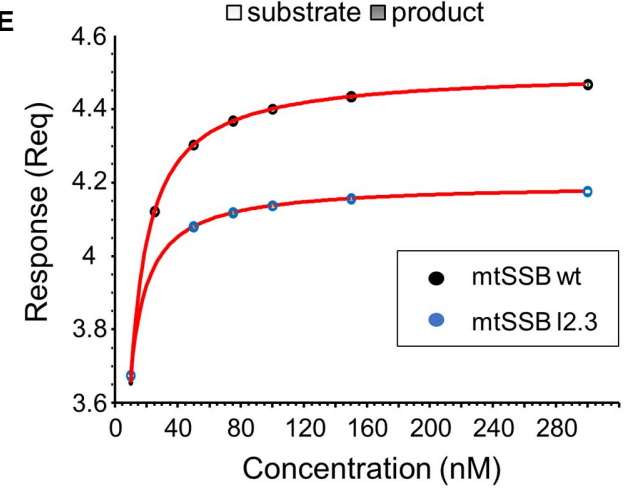

FIGURE 4 | The lack of loop 2.3 impedes the ability of Pol $\gamma$ to displace mtSSB. (A) The processive DNA synthesis assay was performed as described in Figure $3 \mathbf{A}$ except that the $\mathrm{mtSSB}_{12.3}$ variant was used instead of the wild-type mtSSB where indicated. (B) The processivity assay was performed as described under Materials and Methods, using singly-primed M13 DNA, Pol $\gamma \alpha$, and increasing concentrations of the wild-type or mtSSB ${ }_{12.3}$ variant, at the indicated molar ratios to available binding sites on the DNA substrate (mtSSB/DNA). Products of reactions were analyzed by denaturing polyacrylamide gel electrophoresis. (C) The binding affinity of $\mathrm{mtSSB}_{12.3}$ for Pol $\gamma \alpha$ was determined by BLI, as described in Figure 2B, using two sets of association responses upon increasing Pol $\gamma \alpha$ concentrations (indicated), fitted to a 1:1 binding model. (D) The ability of Pol $\gamma \alpha$ to displace wild-type or loop2.3-deficient mtSSB was assessed by primer extension assay on a primer-template (15/59) DNA substrate, at the indicated molar ratio of mtSSB variant to available binding sites on the DNA substrate (mtSSB/DNA). The products were analyzed by native polyacrylamide gel electrophoresis. The relative abundance of the fully double-stranded product was estimated by densitometry and the mean of three independent experiments $( \pm S D)$ is presented in the graph below. (E) ssDNA-binding affinity of the wild-type and mtSSB ${ }_{12.3}$ variant was measured by biolayer interferometry, as described in Materials and Methods. The association traces indicated a non-1:1 binding and mass transfer interference. The DNA-binding affinities were estimated, therefore, from the equilibrium responses of the mass transfer fits (Supplementary Figure 5).

This suggests that in the absence of the repulsive loop 2.3, mtSSB halts the advancement of Pol $\gamma \alpha$ when bound to a downstream stretch of ssDNA, increases its dissociation and consequently, substrate turnover. In the case of the holoenzyme, the effect of $\mathrm{mtSSB}_{\mathrm{l} 2.3}$ was inhibitory to both the efficiency and processivity of exonucleolysis, as in the case of the wild-type mtSSB. 


\section{DISCUSSION}

In this study, we aimed to evaluate the physical interaction between mtSSB and Pol $\gamma$, and demonstrated for the first time that $\mathrm{mtSSB}$ interacts physically with Pol $\gamma \alpha$. The fact that this interaction occurs in the absence of a C-terminal domain, which in cases of both the homologous SSB protein of E. coli or non-homologous SSBs of phages T7 and T4, is necessary to bind cognate DNA polymerases (Oliveira and Ciesielski, 2021), makes our finding unique. Notably, the interaction occurs only in the absence of the accessory subunit Pol $\gamma \beta$. In agreement with this observation, we reported previously that under similar experimental conditions, the binding affinity between the two Pol $\gamma$ subunits $\left(K_{D} 20 \mathrm{nM}\right)$ is over two-fold stronger than that which we report here for the Pol $\gamma \alpha$-mtSSB interaction (Ciesielski et al., 2016a). In the other replication systems, binding of the accessory subunit enhances the interaction of polymerases with SSB proteins, which in turn increases the efficiency of DNA synthesis (Kelman et al., 1998; Salinas and Benkovic, 2000; Hernandez and Richardson, 2019). This contrasts with the case of the mitochondrial counterparts, as we observed that the affinity for mtSSB binding is inversely related with the efficiency of DNA synthesis, especially at high mtSSB concentrations (Figure 3D; Supplementary Figure 4). These differences imply that polymerase- SSB interactions in different biological systems may have distinct physiological roles.

Pursuing the functional relevance of the mtSSB-Pol $\gamma \alpha$ interaction, we found that $\mathrm{mtSSB}$ facilitates loading of Pol $\gamma \alpha$ onto short gap sites, thereby increasing the efficiency of primer excision (Figure 3D). This, together with the exclusive ability of Pol $\gamma \alpha$ (i.e., but not holoenzyme) to excise 8-oxoguanine (Wong et al., 2009), implies that the interaction with $\mathrm{mtSSB}$ may facilitate the engagement of Pol $\gamma \alpha$ in excision-based mtDNA repair mechanisms. Such mechanisms involve a gap-filling step, and although we found that mtSSB had no effect on this activity of Pol $\gamma \alpha$, the higher fidelity of Pol $\gamma \alpha$, together with its inability in strand displacement (Figure 3C), argue that it may serve in this capacity as well. Conversely, in addition to its inability to excise short gaps, Pol $\gamma$ holoenzyme exhibits lower fidelity, and relatively-effective strand displacement synthesis, which generates long non-ligatable 5 '-flaps that are deleterious to mtDNA replication (Macao et al., 2015). Notably, the in vivo expression levels of the proteins under study (Figure 2E) suggest that Pol $\gamma \alpha$ is present in human tissues at a level sufficient to perform the putative repair function, in addition to its role as the component of the replisome. Interestingly, the fact that primer excision was stimulated by mtSSB only in the absence of a sufficiently-long binding site for it on the DNA substrate might suggest that the putative repair occurs post-replication, when mtSSB binding sites would likely be limited, thus resulting in a pool of mtSSB available to load Pol $\gamma \alpha$ onto short gaps. On the other hand, binding of $\mathrm{mtSSB}$ to the primer-template did not stimulate the exonucleolytic activity of Pol $\gamma \alpha$ and inhibited that of the holoenzyme, which, taken together with the fact that binding of mtSSB to ssDNA template stimulates processive DNA synthesis (Figure 3A), suggests that the presence of mtSSB on the DNA template favors mtDNA replication over repair/ excision functions, which has potential implications for mitochondrial/cellular regulation.

We proposed earlier that negatively-charged loop 2.3 moieties extrude from the surface of the mtSSB tetramer to face the advancing Pol $\gamma$ (Figure 1), and mediate a repulsive interaction that drives the unwrapping and displacement of mtSSB from the DNA template (Cerrón et al., 2019). This putative mechanism is supported by the two-fold increase in the Pol $\gamma \alpha$-mtSSB binding affinity upon loop 2.3 deletion. The proposed mechanism also assumes that a lack of loop 2.3 diminishes the repulsive character of the interaction, thereby decreasing the efficiency of mtSSB displacement, which also agrees with our observations (Figure 4D). The relevance of the repulsive interaction between mtSSB and Pol $\gamma$ for mtDNA synthesis is also corroborated by the inhibitory effect of high salt concentrations on Pol $\gamma$ activity that we demonstrated previously (Oliveira and Kaguni, 2011). High ionic strength is likely to eliminate the electrostatic repulsion between the two proteins, and in turn disable the ability of Pol $\gamma$ to displace mtSSB. We also observed that the presence of loop 2.3 weakens the DNA binding activity of $\mathrm{mtSSB}$, as in its absence the affinity of mtSSB for ssDNA increases $\sim 2$-fold (Figure 4E). This effect can again be attributed to the negative charge of the loop, which may be repulsive to the negatively-charged DNA strand. Tighter binding to DNA by $\mathrm{mtSSB}_{12.3}$ may contribute to the reduced ability of Pol $\gamma \alpha$ to displace it (Figures 4B,D).

The reduced capability to displace $\mathrm{mtSSB}$ upon deletion of loop 2.3 may be the direct cause of the elevated efficiency of Pol $\gamma \alpha$ activity in the processive DNA synthesis assay (Figure 4A). We observed that the stimulatory effect of mtSSB occurs only when the DNA template contains long stretches of ssDNA that may allow non-productive binding of Pol $\gamma \alpha$ (Figures 3A,B). In contrast, we observed no stimulation of Pol $\gamma \alpha$ activity in assays using DNA substrates that allow only productive binding of Pol $\gamma \alpha$ (Figures 3C, 4D). Taken together, these observations argue that mtSSB increases the activity of Pol $\gamma \alpha$ by restricting its non-productive residence on unprimed ssDNA stretches, thereby increasing the frequency of the primer binding. Deletion of loop 2.3 may enhance this mechanism, as $\mathrm{mtSSB}_{12.3}$ limits the advancement of Pol $\gamma \alpha$ more efficiently than the wild-type protein (Figures 4B,D), which in turn could promote DNA substrate turnover. This is supported directly by our finding that the processivity of the exonucleolytic activity of Pol $\gamma \alpha$ in the presence of downstream-bound $\mathrm{mtSSB}_{12.3}$ is reduced to a single nucleotide, while the overall abundance of exonucleolytic products remains unchanged (Figure 3D).

\section{DATA AVAILABILITY STATEMENT}

The original contributions presented in the study are included in the article/Supplementary Material; further inquiries can be directed to the corresponding authors. 


\section{AUTHOR CONTRIBUTIONS}

LK and GC designed the experimental approach and analyzed the data. GC executed the biolayer interferometry experiments and processivity assays, and drafted the manuscript. SK and CB conducted gel-based assays. LK provided critical comments and edited the manuscript. All authors contributed to the article and approved the submitted version.

\section{FUNDING}

GC was supported partially by a grant from the Auburn University at Montgomery Research Grant-in-Aid Program, and by funding from the National Institutes of Health (GM45295) and the Academy of Finland to LK. CB was supported by the Undergraduate Research \& Creative Activity grant from Auburn University at Montgomery. The UAB VSRC cores are supported by NIH grant P30 EY003039.

\section{REFERENCES}

Cerrón, F., de Lorenzo, S., Lemishko, K. M., Ciesielski, G. L., Kaguni, L. S., Cao, F. J., et al. (2019). Replicative DNA polymerases promote active displacement of SSB proteins during lagging strand synthesis. Nucleic Acids Res. 47, 5723-5734. doi: 10.1093/nar/gkz249

Ciesielski, G. L., Bermek, O., Rosado-Ruiz, F. A., Hovde, S. L., Neitzke, O. J., Griffith, J. D., et al. (2015). Mitochondrial single-stranded DNA-binding proteins stimulate the activity of DNA polymerase $\gamma$ by organization of the template DNA. J. Biol. Chem. 290, 28697-28707. doi: 10.1074/jbc.M115.673707

Ciesielski, G. L., Hytönen, V. P., and Kaguni, L. S. (2016a). Biolayer interferometry: a novel method to elucidate protein-protein and protein-DNA interactions in the mitochondrial DNA replisome. Methods Mol. Biol. 1351, 223-231. doi: 10.1007/978-1-4939-3040-1_17

Ciesielski, G. L., Oliveira, M. T., and Kaguni, L. S. (2016b). Animal mitochondrial DNA replication. Enzyme 39, 255-292. doi: 10.1016/bs.enz.2016.03.006

Curth, U., Genschel, J., Urbanke, C., and Greipel, J. (1996). In vitro and in vivo function of the C-terminus of Escherichia coli single-stranded DNA binding protein. Nucleic Acids Res. 24, 2706-2711. doi: 10.1093/nar/24.14.2706

Gray, H., and Wong, T. W. (1992). Purification and identification of subunit structure of the human mitochondrial DNA polymerase. J. Biol. Chem. 267, 5835-5841. doi: 10.1016/S0021-9258(18)42629-4

He, Q., Shumate, C. K., White, M. A., Molineux, I. J., and Yin, Y. W. (2013). Exonuclease of human DNA polymerase gamma disengages its strand displacement function. Mitochondrion 13, 592-601. doi: 10.1016/j. mito.2013.08.003

Hernandez, A. J., and Richardson, C. C. (2019). Gp2.5, the multifunctional bacteriophage T7 single-stranded DNA binding protein. Semin. Cell Dev. Biol. 86, 92-101. doi: 10.1016/j.semcdb.2018.03.018

Hori, K., Mark, D. F., and Richardson, C. C. (1979). Deoxyribonucleic acid polymerase of bacteriophage T7. Purification and properties of the phageencoded subunit, the gene 5 protein. J. Biol. Chem. 254, 11591-11597. doi: 10.1016/S0021-9258(19)86526-2

Johnson, A. A., Tsai, Y., Graves, S. W., and Johnson, K. A. (2000). Human mitochondrial DNA polymerase holoenzyme: reconstitution and characterization. Biochemistry 39, 1702-1708. doi: 10.1021/bi992104w

Kaguni, L. S. (2004). DNA polymerase gamma, the mitochondrial replicase. Annu. Rev. Biochem. 73, 293-320. doi: 10.1146/annurev. biochem.72.121801.161455

Kazak, L., Reyes, A., and Holt, I. J. (2012). Minimizing the damage: repair pathways keep mitochondrial DNA intact. Nat. Rev. Mol. Cell Biol. 13, 659-671. doi: 10.1038/nrm3439

Kelman, Z., Yuzhakov, A., Andjelkovic, J., and O’Donnell, M. (1998). Devoted to the lagging strand-the subunit of DNA polymerase III holoenzyme contacts

\section{ACKNOWLEDGMENTS}

GC would like to thank Szymon Ciesielski (University of Wisconsin) for help with molecular modeling. We also thank Vesa Hytönen (University of Tampere) for advice and useful discussions of the biolayer interferometry data. We also thank the University of Alabama at Birmingham, Vision Science Research Center (UAB VSRC), Molecular \& Cellular Analysis Core for allowing us to use their GE Typhoon Trio+ Variable Mode Imager for quantification of fluorescent signals on polyacrylamide gels.

\section{SUPPLEMENTARY MATERIAL}

The Supplementary Material for this article can be found online at: https://www.frontiersin.org/articles/10.3389/fgene.2021.7218 64/full\#supplementary-material

SSB to promote processive elongation and sliding clamp assembly. $E M B O$ J. 17, 2436-2449. doi: 10.1093/emboj/17.8.2436

Korhonen, J. A., Pham, X. H., Pellegrini, M., and Falkenberg, M. (2004). Reconstitution of a minimal mtDNA replisome in vitro. EMBO J. 23, 2423-2429. doi: 10.1038/sj.emboj.7600257

Lee, Y. S., Lee, S., Demeler, B., Molineux, I. J., Johnson, K. A., and Yin, Y. W. (2010). Each monomer of the dimeric accessory protein for human mitochondrial DNA polymerase has a distinct role in conferring processivity. J. Biol. Chem. 285, 1490-1499. doi: 10.1074/jbc.M109.062752

Longley, M. J., Nguyen, D., Kunkel, T. A., and Copeland, W. C. (2001). The fidelity of human DNA polymerase gamma with and without exonucleolytic proofreading and the p55 accessory subunit. J. Biol. Chem. 276, 38555-38562. doi: 10.1074/jbc.M105230200

Longley, M. J., Prasad, R., Srivastava, D. K., Wilson, S. H., and Copeland, W. C. (1998a). Identification of 5'-deoxyribose phosphate lyase activity in human DNA polymerase gamma and its role in mitochondrial base excision repair in vitro. Proc. Natl. Acad. Sci. U. S. A. 95, 12244-12248. doi: 10.1073/pnas.95.21.12244

Longley, M. J., Ropp, P. A., Lim, S. E., and Copeland, W. C. (1998b). Characterization of the native and recombinant catalytic subunit of human DNA polymerase gamma: identification of residues critical for exonuclease activity and dideoxynucleotide sensitivity. Biochemistry 37, 10529-10539. doi: 10.1021/bi980772w

Macao, B., Uhler, J. P., Siibak, T., Zhu, X., Shi, Y., Sheng, W., et al. (2015). The exonuclease activity of DNA polymerase $\gamma$ is required for ligation during mitochondrial DNA replication. Nat. Commun. 6:7303. doi: 10.1038/ ncomms 8303

McHenry, C., and Kornberg, A. (1977). DNA polymerase III holoenzyme of Escherichia coli. Purification and resolution into subunits. J. Biol. Chem. 252, 6478-6484. doi: 10.1016/S0021-9258(17)39983-0

Morin, J. A., Cerrón, F., Jarillo, J., Beltran-Heredia, E., Ciesielski, G. L., Arias-Gonzalez, J. R., et al. (2017). DNA synthesis determines the binding mode of the human mitochondrial single-stranded DNA-binding protein. Nucleic Acids Res. 45, 7237-7248. doi: 10.1093/nar/gkx395

Oliveira, M. T., and Ciesielski, G. L. (2021). The essential, ubiquitous singlestranded DNA-binding proteins. Methods Mol. Biol. 2281, 1-21. doi: 10.1007/978-1-0716-1290-3_1

Oliveira, M. T., and Kaguni, L. S. (2009). Comparative purification strategies for Drosophila and human mitochondrial DNA replication proteins: DNA polymerase gamma and mitochondrial single-stranded DNA-binding protein. Methods Mol. Biol. 554, 37-58. doi: 10.1007/978-1-59745-521-3_3

Oliveira, M. T., and Kaguni, L. S. (2011). Reduced stimulation of recombinant DNA polymerase $\gamma$ and mitochondrial DNA (mtDNA) helicase by variants of mitochondrial single-stranded DNA-binding protein (mtSSB) correlates with defects in mtDNA replication in animal cells. J. Biol. Chem. 286, 40649-40658. doi: 10.1074/jbc.M111.289983 
Pinz, K. G., and Bogenhagen, D. F. (2006). The influence of the DNA polymerase gamma accessory subunit on base excision repair by the catalytic subunit. DNA Repair 5, 121-128. doi: 10.1016/j.dnarep.2005.08.014

Piro-Mégy, C., Sarzi, E., Tarrés-Solé, A., Péquignot, M., Hensen, F., Quilès, M., et al. (2019). Dominant mutations in mtDNA maintenance gene SSBP1 cause optic atrophy and foveopathy. J. Clin. Invest. 30, 143-156. doi: 10.1172/ JCI128513

Raghunathan, S., Kozlov, A. G., Lohman, T. M., and Waksman, G. (2000). Structure of the DNA binding domain of E. coli SSB bound to ssDNA. Nat. Struct. Biol. 7, 648-652. doi: 10.1038/77943

Salinas, F, and Benkovic, S. J. (2000). Characterization of bacteriophage T4coordinated leading- and lagging-strand synthesis on a minicircle substrate. Proc. Natl. Acad. Sci. U. S. A. 97, 7196-7201. doi: 10.1073/pnas.97.13.7196

Stumpf, J. D., Saneto, R. P., and Copeland, W. C. (2013). Clinical and molecular features of POLG-related mitochondrial disease. Cold Spring Harb. Perspect. Biol. 5:a011395. doi: 10.1101/cshperspect.a011395

Szymanski, M. R., Kuznetsov, V. B., Shumate, C., Meng, Q., Lee, Y. S., Patel, G., et al. (2015). Structural basis for processivity and antiviral drug toxicity in human mitochondrial DNA replicase. EMBO J. 34, 1959-1970. doi: 10.15252/ embj. 201591520

Thul, P. J., Åkesson, L., Wiking, M., Mahdessian, D., Geladaki, A., Ait Blal, H., et al. (2017). A subcellular map of the human proteome. Science 356:eaal3321. doi: $10.1126 /$ science.aal3321

Uhlen, M., Fagerberg, L., Hallström, B. M., Lindskog, C., Oksvold, P., Mardinoglu, A., et al. (2015). Proteomics. Tissue-based map of the human proteome. Science 347:1260419. doi: 10.1126/science.1260419

Uhlen, M., Zhang, C., Lee, S., Sjöstedt, E., Fagerberg, L., Bidkhori, G., et al. (2017). A pathology atlas of the human cancer transcriptome. Science 357:eaan2507. doi: $10.1126 /$ science.aan 2507
Wernette, C. M., Conway, M. C., and Kaguni, L. S. (1988). Mitochondrial DNA polymerase from Drosophila melanogaster embryos: kinetics, processivity, and fidelity of DNA polymerization. Biochemistry 27, 6046-6054. doi: 10.1021/ bi00416a033

Wong, T. S., Rajagopalan, S., Townsley, F. M., Freund, S. M., Petrovich, M., Loakes, D., et al. (2009). Physical and functional interactions between human mitochondrial single-stranded DNA-binding protein and tumour suppressor p53. Nucleic Acids Res. 37, 568-581. doi: 10.1093/nar/gkn974

Yang, C., Curth, U., Urbanke, C., and Kang, C. (1997). Crystal structure of human mitochondrial single-stranded DNA binding protein at 2.4 A resolution. Nat. Struct. Biol. 4, 153-157. doi: 10.1038/nsb0297-153

Conflict of Interest: The authors declare that the research was conducted in the absence of any commercial or financial relationships that could be construed as a potential conflict of interest.

Publisher's Note: All claims expressed in this article are solely those of the authors and do not necessarily represent those of their affiliated organizations, or those of the publisher, the editors and the reviewers. Any product that may be evaluated in this article, or claim that may be made by its manufacturer, is not guaranteed or endorsed by the publisher.

Copyright (c) 2021 Ciesielski, Kim, de Bovi Pontes and Kaguni. This is an open-access article distributed under the terms of the Creative Commons Attribution License (CC BY). The use, distribution or reproduction in other forums is permitted, provided the original author(s) and the copyright owner(s) are credited and that the original publication in this journal is cited, in accordance with accepted academic practice. No use, distribution or reproduction is permitted which does not comply with these terms. 